\title{
MATCHED TRANSFORMERS FOR SYNCHRO AND RESOLVER APPLICATIONS
}

\author{
M. PRATT \\ Professional Components Division, Ferranti Ltd., Dundee, UK
}

(Received December 17; 1974; in final form March 3, 1975)

\begin{abstract}
Transformer pairs in Scott Tee and similar transformer arrangements have been used for some considerable time in precision synchro/resolver angular measuring gear and in synchro to digital converters. Literature on the subject of transformer requirements is, however, scant or non-existent.1,2 This paper describes the basic principle of transformer operation and develops a design approach which, although aimed primarily at the minimisation of transformer hardware size, still maintains the required level of angular accuracy. The method applies to transformers utilised in mobile systems, particularly transformer arrangements working under loaded conditions.
\end{abstract}

\section{INTRODUCTION}

Synchro shaft angle to digital conversion techniques are used extensively in airborne and shipborne systems to give direct read-out positional data on synchro and resolver elements with an ability to reposition synchro devices from a central control computer.

Shaft angle data, to and from the control point, is by a three wire system, usually with the synchro elements energised at a frequency of $400 \mathrm{~Hz}$.

For accurate translation of the positional data, it is convenient to perform a three wire to four wire conversion to produce two output signals proportional to the sine and cosine of the synchro angular position. By suitable process methods, a single analogue signal can be obtained which is readily converted to the equivalent digital angle.

While various conversion methods can be used, it can be demonstrated that the incorporation of specially matched transformers in the synchro to digital loop will, in addition to a general simplification of the converter design, give added benefits in the way of:

(a) Complete isolation, input to output.

(b) A high degree of stability against extreme climatic and temperature variation.

(c) An ability to withstand sustained overloading with general overall rugged characteristics unsurpassed by other components.

Mobile applications demand a proper design and manufacturing approach leading to minimised weight and volume, especially in transformers looking towards and positioning the synchro. (Digital to synchro mode application.)

\section{THREE TO FOUR WIRE CONVERSION}

The use of transformers in three to four wire conversion is readily understood by first considering Figure 1. which is descriptive of a synchro element connected to a pair of transformers in an arrangement known as the Scott Tee connection. This transformer connection is used basically in power applications to give three phase to two phase conversion. Used as shown, the line-to-line voltage outputs from the synchro will be in the form:

$$
\begin{aligned}
& V_{13}=E_{s} \sin \theta \\
& V_{23}=E_{s} \sin \left(\theta+120^{\circ}\right) \\
& V_{12}=E_{s} \sin \left(\theta+240^{\circ}\right)
\end{aligned}
$$

where $E_{s}$ is the maximum voltage across any two lines. and $\theta$ is the synchro electrical angle.

It should be noted that the value $E_{s}$ can be written in the instantaneous form $e_{s} \sin \omega t$ and is related to the voltage induced in the three stator coils from the energised rotor coil. For simplicity, however, only the peak voltage values need be considered.

$V_{23}$ and $V_{12}$ can be re-arranged to give:

$$
V_{23}=E_{s}\left(-1 / 2 \sin \theta+\frac{3^{1 / 2}}{2} \cos \theta\right)
$$




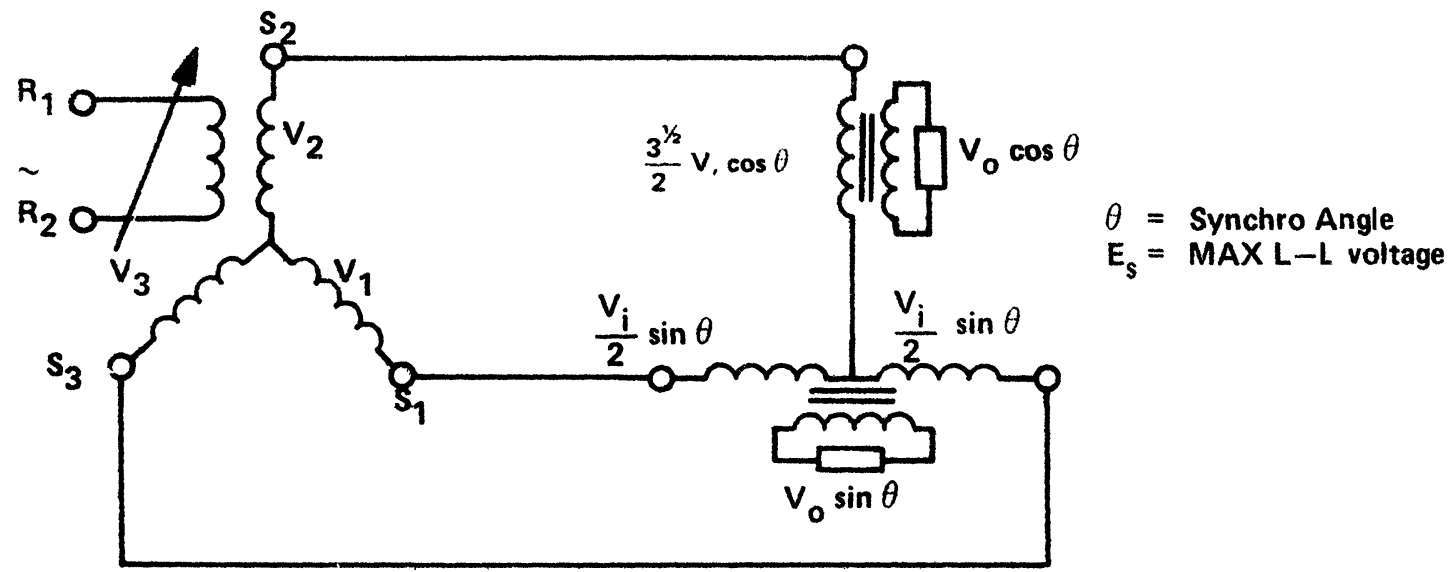

FIGURE 1 Synchro-to-transformer connections.

$$
V_{12}=E_{s}\left(-1 / 2 \sin \theta-\frac{3^{1 / 2}}{2} \cos \theta\right)
$$

The volts across the individual synchro stator coils will be:

$$
\begin{aligned}
& V_{2}=\frac{E_{s}}{3^{1 / 2}} \cos \theta \\
& V_{1}=\frac{E_{s}}{3^{1 / 2}}\left(-1 / 2 \cos \theta-\frac{3^{1 / 2}}{2} \sin \theta\right)
\end{aligned}
$$

and

$$
V_{3}=\frac{E_{s}}{3^{1 / 2}}\left(-1 / 2 \cos \theta+\frac{3^{1 / 2}}{2} \sin \theta\right)
$$

Writing down the volts across each half of the centre tapped transformer as

$$
V_{C T}=1 / 2\left(V_{1}+V_{3}\right)
$$

and substituting for $V_{1}$ and $V_{3}$ from Eqs. (1) and (2) gives

$$
V_{C T}=-\frac{E_{S}}{2} \frac{\operatorname{Cos} \theta}{3^{1 / 2}}
$$

Further consideration of the volts across the untapped transformer will indicate this in the form:

$$
\begin{aligned}
V_{C T 2} & =\frac{E_{s}}{3^{1 / 2}} \operatorname{Cos} \theta+\frac{E_{s}}{2.3^{1 / 2}} \operatorname{Cos} \theta \\
& =\frac{3^{1 / 2}}{2} E_{s} \operatorname{Cos} \theta
\end{aligned}
$$

Also

$$
\begin{aligned}
V_{C T 2} & =\frac{E_{s}}{3^{1 / 2}} \frac{3^{1 / 2}}{2} \sin \theta \\
& =\frac{E_{s}}{2} \sin \theta
\end{aligned}
$$

and

$$
\begin{aligned}
V_{C T 1} & =\frac{E_{s}}{3^{1 / 2}} \frac{3^{1 / 2}}{2} \sin \theta \\
& =-\frac{E_{s}}{2} \sin \theta
\end{aligned}
$$

From this it will be seen that the signal voltage across the whole of the tapped transformer behaves according to the sine of the synchro angle, while that across the untapped transformer behaves according to the 
cosine of the synchro angle. The volts amplitude of the latter is, however, $3 \frac{1}{2} / 2$ times that of the tapped transformer.

With an equal turns per volt relationship on each transformer, output windings of equal turns will give output voltages varying with the respective inputs and equal to each other in maximum amplitude.

\section{ERRORS AND ERROR CAUSES}

The two main sources of system error are

(a) Errors through the transformers

(b) Errors due to the transformers loading the synchro element.

Investigation of error mechanism, allied with a knowledge of synchro action, will indicate that minimisation of most loading errors, both through the transformers and back to the synchro element, are directly related to the degree of balance obtainable in the series and shunt impedance values.

\subsection{Transformation Errors}

Considering errors through the transformers, the two main contributory parameters are

(a) Transformation voltage ratio

(b) Series winding impedance
Consideration of transformation errors, related to the mechanism of four wire coversion, will indicate that the voltage excitation across the transformers in the Scott pair, as already indicated, is in a differential mode, giving a coincidence of maximum and minimum core flux change in each unit. Only at the mid points (45 degrees, 135 degrees etc) in each quadrant of synchro travel will the excitation be equal. This is illustrated graphically in Figure 2.

The effect of this variable excitation will be to vary the apparent voltage transformation ratio through the transformers, the voltage transformation being the actual measured input to output voltage ratio as against the physical turns ratio value of each transformer. Related to the mid quadrant points, the tendency is to give an apparent decrease in the ratio as the excitation decreases and an increase in the ratio towards the high excitation points.

If angular errors through the transformers are plotted over the first two quadrants of synchro travel, by extracting the ratio of the two outputs as the tangent of the angle over the first and third octants and the co-tangent of the angle over the second and fourth octants, the error factor under classic conditions will be as illustrated in Figure 3.

Examination of this error form will indicate that, considered from the 45 degree and 135 degree balanced points, the resultant errors will bear a direct relationship to the variation of the effective transformation ratio through both transformers in the pair.

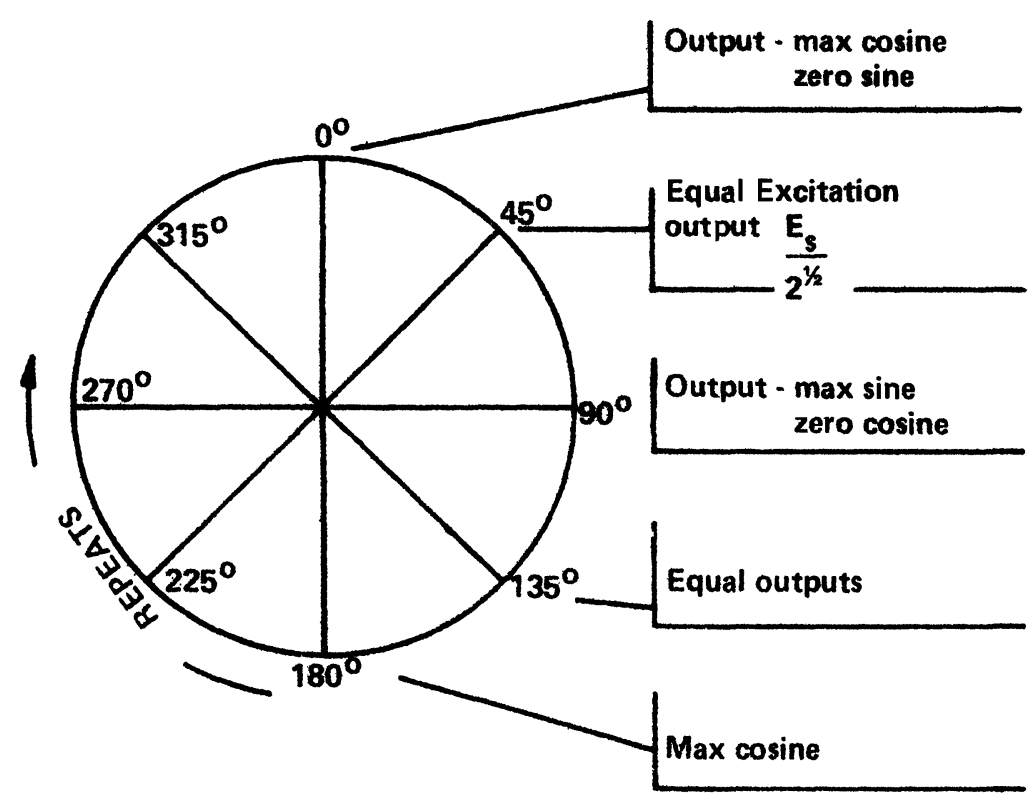

FIGURE 2 Differential voltage distribution. 

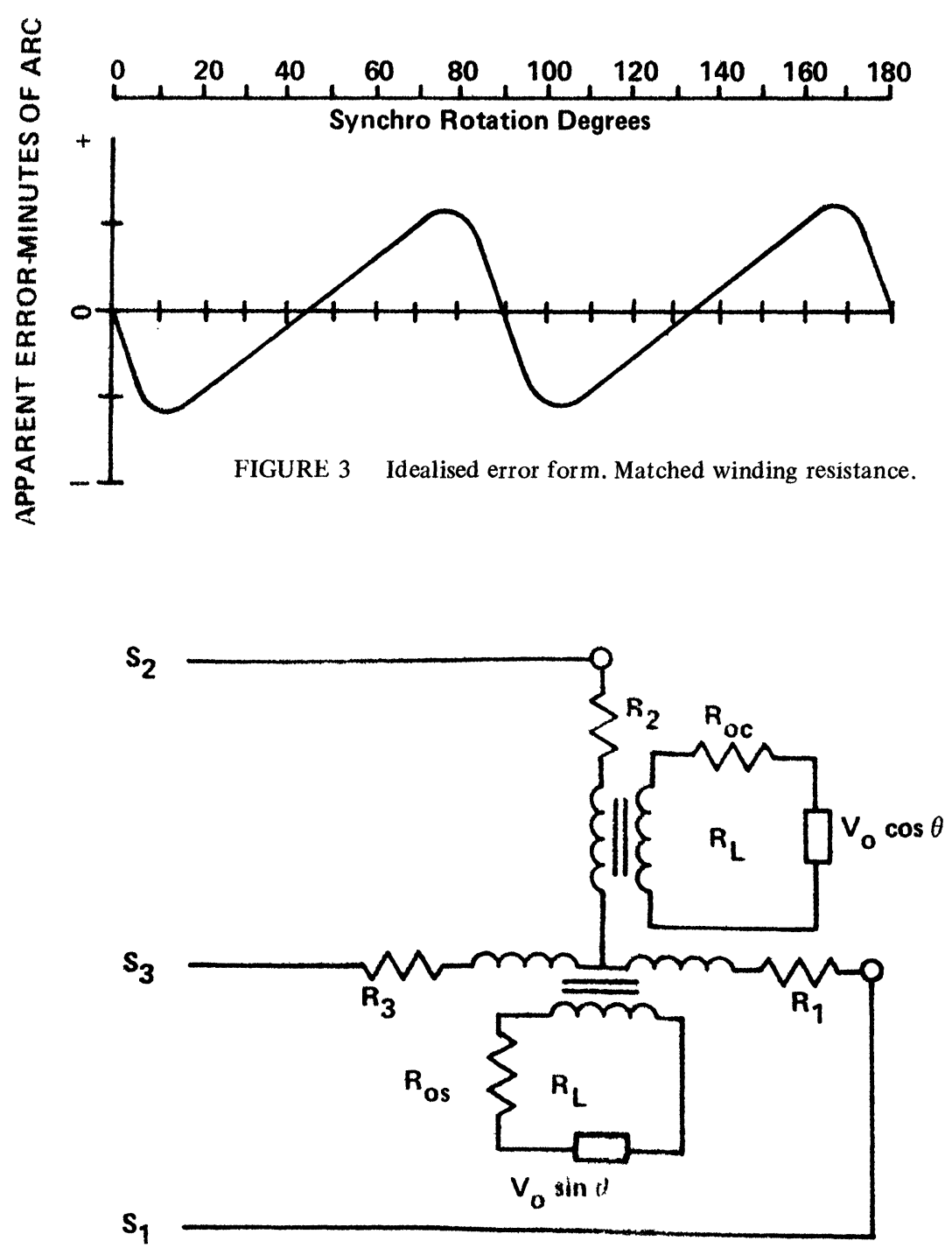

FIGURE 4 Equivalent circuit showing winding and load resistances.

\subsection{Loading Errors though the Transformers}

The variation of output terminal voltage on each transformer and the variation of the voltage ratio between transformers, with the application of loads across the output, will be dependant on the value of transformer series winding impedance and the value of the applied loads.

Consider Figure 4. which represents the equivalent circuit of a Scott Tee transformer pair loaded on both outputs with matched resistors $R_{L}$. Resistors $R_{o s}$ and
$R_{o c}$ are the output winding resistances of the sine and cosine transformers, $R_{1}$ and $R_{3}$ the input resistance of both halves of the sine transformed and $R_{2}$ the input winding resistance of the cosine transformer.

Referring all values to the appropriate output windings will give:

$$
\begin{aligned}
R(\sin ) & =R_{1}^{\prime}+R_{3}^{\prime}+R_{o s} \\
& =R_{s}^{\prime}+R_{o s}
\end{aligned}
$$




$$
\begin{aligned}
R(\cos ) & =\frac{4}{3}\left(R_{2}^{\prime}+\frac{R_{1}^{\prime} R_{3}^{\prime}}{R_{1}^{\prime}+R_{3}^{\prime}}\right)+R_{o c} \\
& =\frac{4}{3}\left(R_{2}^{\prime}+\frac{R_{s}^{\prime}}{4}\right)+R_{o c}
\end{aligned}
$$

where $R_{s}^{\prime}=R_{1}^{\prime}+R_{2}^{\prime}=$ Total input sine resistance referred to the output winding.

$$
\begin{aligned}
R_{2}^{\prime}= & \text { Input cosine resistance } \\
& \text { referred to the output } \\
& \text { winding. }
\end{aligned}
$$

The effective voltage at each output will be:

$$
V_{o}(\sin )=K E_{s}^{\prime} \sin \theta-I_{o} \sin \theta\left(R_{s}^{\prime}+R_{o s}\right)
$$

and

$$
V_{o}(\cos )=K E_{s}^{\prime} \cos \theta-I_{o} \cos \theta\left[\frac{4}{3}\left(R_{2}^{\prime}+\frac{R_{s}^{\prime}}{4}\right)+R_{o c}\right]
$$

The first term in the expression is the voltage due to transformation through the transformers; the second term is the voltage drop caused by the load current $I_{o}$ through the total series winding resistance on each transformer.

At the mid quadrant points, both outputs will be equal as will each term in the equation.

Equating the voltage drop values gives:-

$$
I_{o} \sin \theta\left(R_{s}^{\prime}+R_{o s}\right)=I_{o} \cos \theta \frac{4}{3}\left(R_{2}^{\prime}+\frac{R_{s}^{\prime}}{4}+R_{o c}\right)
$$

or

$$
R_{s}^{\prime}+R_{o s}=\frac{4}{3}\left(R_{2}^{\prime}+\frac{R_{s}^{\prime}}{4}\right)+R_{o c}
$$

if

$$
R_{o s}=R_{o c}
$$

then

$$
R_{2}^{\prime}=\frac{R_{s}^{\prime}}{2}
$$

In other words, under load conditions, equal output voltages are achieved at the mid quadrant points, when the input winding resistance of the cosine transformer.is half the value of the sine transformer input winding resistance, both output resistance values being equal. Further investigation will indicate that for complete balance $R_{1}^{\prime}$, must be equal to $R_{3}^{\prime}$.

\subsection{Transformer Series Impedance Limits}

Consideration of series impedance effects will indi- cate that the output voltage balance can be obtained under two distinct conditions, i.e.

(a) Completely matched winding and load conditions.

(b) Winding resistance values tending towards zero.

The latter condition implies a unit tending to be infinitely large and, therefore, unacceptable in compact systems under consideration.

With imposed requirements of matched winding resistance, it is clear that a method must be derived to lay down limits of allowable winding resistance variation between the transformers in the pair and between the windings on each transformer.

Writing down the output volts in terms of winding resistance and load resistance will give:-

$$
V_{o}(\sin )=E_{s}^{\prime}\left[K \sin \theta-\sin \theta \frac{\left(R_{s}^{\prime}+R_{o s}\right)}{R_{L}}\right]
$$

and

$V_{o}(\cos )=E_{s}^{\prime}\left[K \cos \theta-\cos \theta \frac{4}{3 R_{L}}\left(R_{2}^{\prime}+\frac{R_{s}^{\prime}}{4}\right)+\frac{R_{o c}}{R_{L}}\right]$

The output voltage ratio at 45 degrees can be written as:-

$$
\frac{V_{o}(\sin )}{V_{o}(\cos )}=V_{o}(\tan )=\frac{1-\frac{R_{s}^{\prime}+R_{o s}}{R_{L}}}{1-\frac{4}{3 R_{L}}\left(R_{2}^{\prime}+\frac{R_{s}^{\prime}}{4}\right)+\frac{R_{o c}}{R_{L}}}=F
$$

where $\mathrm{F}$ is a factor related to the degree of balance. If the equality of winding resistance between the transformers is maintained, it will be seen that $F=1$. This is equivalent to a transformer pair with zero error at 45 degrees. The expression relating allowable winding resistance variation to given values of error factor F, can be rewritten in various forms. In practice, the allowable resistance variation will require to be set for each winding. The proportion of allowable resistance variation will depend on a number of factors, including the winding form and the relative dimensions of the winding wire diameter on each winding.

\subsection{Winding Resistance Load Resistance Relationships}

Further reasoning on the question of winding resistance balance will indicate that the degree of required balance is dependant on the relative values of winding resistance and load resistance. 


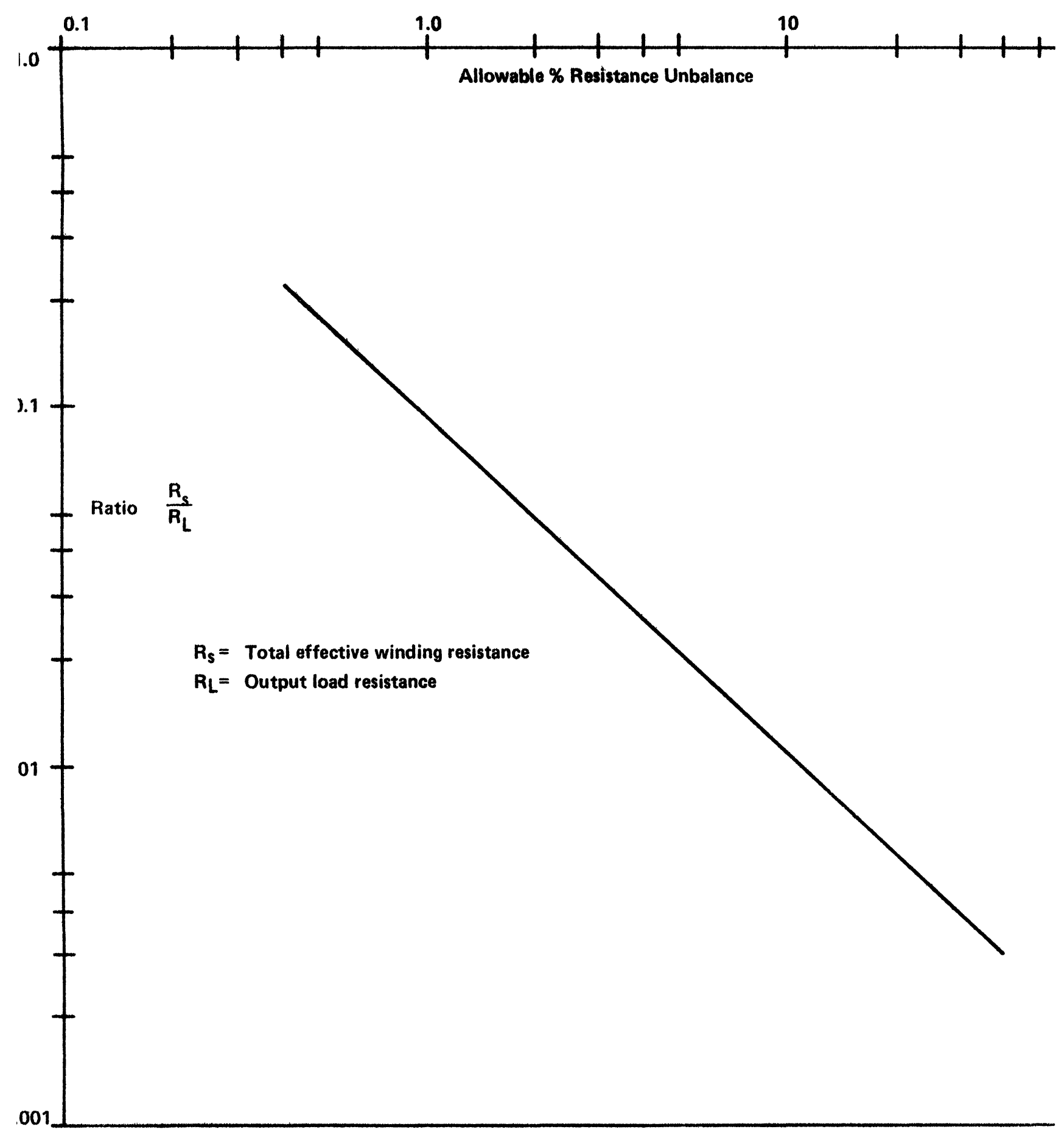

FIGURE 5 Allowable winding resistance mismatch related to output load for an allowable error of $0.6^{\prime}$ at $45^{\circ}$. 
This can best be demonstrated by first considering the transformers on a unity input to output ratio basis and, for a given allowable error, plotting the allowable degree of resistance mismatch between the sine and cosine transformers for various winding to load resistance values. This has been carried out in Figure 5. The vertical scale is in terms of winding resistance/load resistance ratio and the horizontal scale to allowable winding resistance mismatch between units in the Scott pair.

It will be seen that the requirements of winding resistance balance increases as the winding resistance/ load resistance ratio increases. In other words, the allowable tolerance on resistance mismatch diminishes as the winding resistance approaches the value of the load resistance.

The effect of resistance mismatch under loaded conditions is illustrated in Figure 6. The increase and displacement of angular errors from the balanced condition will depend on the degree of mismatch between the sine and cosine resistance values.

Increasing the loading for a given resistance mismatch will give a progressive displacement of the balanced mid quadrant null points out towards the end of the quadrants. This will lead to the extreme case where the maximum error will coincide with the original mid quadrant null point.
It is of interest to note that the same effect will be produced if the effective turns relationship between the input sine and cosine windings is varied about the optimum $3^{1 / 2} / 2$ point. This is to be expected since, in effect, the normal output voltage ratio balance is misplaced.

Other functions involved in the error function will be the phase change through the transformers and the effect of series reactance. In properly designed transformers, both these parameters will be negligibly small. The series reactance will be, generally, more than an order of magnitude down on the resistive element. The effect, even under close tolerance balance, will be fractional.

\subsection{Synchro Loading Errors}

Errors caused by loading effects on the synchro element are due to unbalance in the shunt impedance values of the transformer pair and the line-to-line series impedance unbalance of the synchro element.

Depending on the core arrangement, transformer shunt impedance unbalance can be as high as twelve percent. Scott Tee arrangements will, by virtue of the connection, give unbalanced effects.

The effects can be reduced by resorting to selected core trim and winding trim methods. This, however,

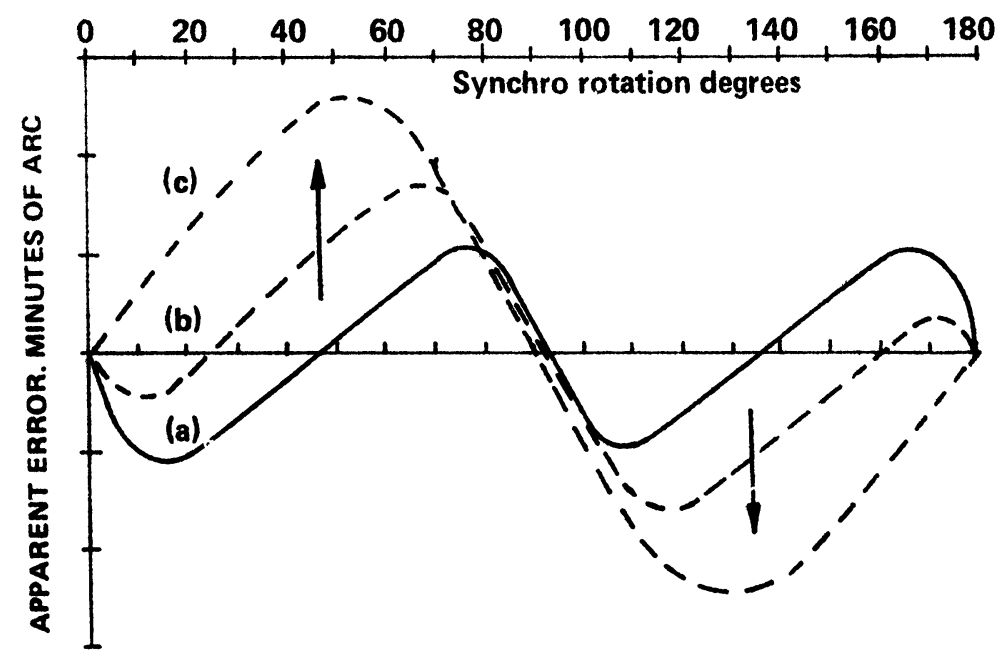

(a) Balanced condition

(b) Progressive error increase

(c) Complete displacement of normal balance null points

FIGURE 6 Increase and displacement of angular errors from minimised condition, due to mismatch of winding resistance. 
is not always a practical solution especially over production runs.

The effect of synchro loading is more effectively reduced by increasing the absolute value of transformer shunt impedance to a point where the effect of any unbalance is minimal.

This may be best understood if both the synchro series impedance and the transformer shunt impedance values are considered in relation to output errors, with a given mismatch between the shunt impedance values.

The impedance presented to the sine transformer will be

$$
2 Z_{s s}
$$

and to the cosine transformer input

$$
\frac{3}{2} Z_{s s}
$$

where $Z_{s s}$ is the series, short circuit, line-to-line impedance of the synchro in the form

$$
R_{s s}+j \omega L_{s s}
$$

The effective voltage across the sine transformer input will be

$$
V_{i}(\sin )=e_{s} \sin \theta \frac{Z_{s}}{Z_{s}+2 Z_{s s}}
$$

and across the cosine input:-

$$
V_{i}(\cos )=\frac{3^{1 / 2}}{2} e_{s} \cos \theta \frac{Z_{c}}{Z_{c}+3 / 2 Z_{s s}}
$$

where $Z_{s}=$ Shunt impedance of the sine transformer

$Z_{c}=$ Shunt impedance of the cosine transformer

$$
=\frac{3}{2} Z_{s}
$$

$Z_{c}$ and $Z_{s}=$ the inductive reactance of the input winding in parallel with the reflected load impedance $R_{L}$

$e_{s}=$ Synchro line-to-line, open circuit voltage.

With the turns ratio of $\left(3^{1 / 2} / 2\right): 1$ on the cosine transformer the expression for the cosine output will be similar in form to the sine output. then

The ratio of the output at the 45 degree point is

$$
\begin{aligned}
\frac{V_{o}(\sin )}{V_{o}(\cos )}=V_{o}(\tan ) & =\frac{Z_{s}^{\prime} Z_{c}^{\prime}+2 Z_{s s}^{\prime} Z_{s}^{\prime}}{Z_{s}^{\prime} Z_{c}^{\prime}+2 Z_{s s}^{\prime} Z_{c}^{\prime}}=F \\
& =\frac{1+\frac{2 Z_{s s}^{\prime}}{Z_{c}^{\prime}}}{1+\frac{2 Z_{s s}^{\prime}}{Z_{s^{\prime}}}}
\end{aligned}
$$

It is obvious from this, that, for a given $Z_{s}^{\prime}$ to $Z_{c}^{\prime}$ unbalance, the error factor $F$ will decrease as the ratio of $Z_{s s}^{\prime}$ to $Z_{s}^{\prime}$ and $Z_{c}^{\prime}$ decreases.

In other words, the higher the shunt impedance for a given synchro series inpedance, the lower will be the error in the presence of given values of shunt impedance unbalance.

It will be seen that, so far as error factor is concerned, the magnitude of the $Z_{s s}^{\prime}$ to $Z_{s}^{\prime}$ ratio, has the same effect between the synchro and the transformers as the magnitude of the winding resistance/ load resistance ratio, has through the transformers.

\section{DESIGN ASPECTS}

Transformers of this type would be required to perform the following basic operations:-

(a) Monitor accurately the angular position of synchro transmitter and receiver combinations (synchro to digital application).

(b) Position synchro elements from a driver amplifier source (digital to synchro application).

(c) Act as isolation devices between synchro or resolver elements.

Reduction of transformer package weight and volume is of prime importance in all mobile applications. This leads to a compromise on the various design parameters.

\subsection{Application Requirements}

Transformers performing in the monitor role look back towards the two outputs into high order resistance loads, usually $10 \mathrm{~K} \Omega$ to $100 \mathrm{~K} \Omega$, at relatively low voltage levels. This will tend to give low winding resistance/load resistance ratio values. In the presence of balanced winding resistance, this leads to a relaxation on the tolerance of the output load resistance values and obviates the need of precision components. The fact that the transformers are shunted across relatively high impedance synchro combinations, however, requires the transformer shunt impedance values to be of a high order.

Conversely, the transformer pair incorporated in a system working towards the synchro and performing positional changes, is loaded effectively with impedance values of some two orders of magnitude below the monitor counterparts. This leads to a reduced tolerance of spread on the unit-to-unit and windingto-winding resistance match. At the same time, because of the low effective impedance of the sine 
and cosine drive amplifiers, the shunt impedance of the transformers is of lesser importance.

Transformers working in the isolation mode will require high values of shunt impedance, with effective load values somewhere between the values of the first two applications.

\subsection{Volume Reduction and Reduction Limitations}

In most close tolerance or precision type transformer applications, the tolerance on the variation of the output voltage due mainly to the variation in the winding resistance, is a major problem. The problem increases with loading on the transformer and would tend to restrict size reduction.

With the present application, the only important factor is the ratio of the two output voltage values, the absolute value of the individual outputs being of minor importance.

It will be recalled that the effective voltage through each transformer consists of a transformation and a voltage drop factor, the output being the difference between the two factors. Further consideration will indicate that, for a given transformation characteristic and matched winding resistance conditions, only the voltage drop factor need be considered in the maintenance of the tolerance of the ratio of the two output voltages.

This suggests that provided the matched resistance condition is maintained, the voltage drop factor, and hence the resistance, can be allowed to increase towards some higher limiting value.

Increase in winding resistance is synonymous with reduction in transformer volume. This is equivalent to stating that, to the first approximation, where the output is dependant on the ratio of two output voltages in a matched transformer pair, size and volume can be decreased to a point where only the practical considerations of the mechanical aspects, mainly associated with the ability to handle fine diameter conductors and maintain a level of winding resistance balance in the presence of small winding diameters, would be the limiting factor.

This is generally true in the case of the monitor mode devices. Even under fairly drastic volume reduction, winding resistance to load resistance values can still be of a low enough order to give workable spread values on the winding resistance. It has to be noted, however, that input magnetising current could have an increasing error effect as the input winding resistance increases.

As far as the driver mode transformers are con- cerned, the low values of load resistance resulting in relatively high orders of winding resistance/load resistance values, limits the reduction of transformer volume to a point dictated by the ability to set realistic and consistent tolerance values on the winding resistance balance.

There is, however, another limiting parameter which is only apparent after reconsidering the effect of differential excitation. It will be recalled that at the various extreme, points in the synchro travel, all the excitation is across one unit only in the pair. Depending on the value of the effective load, a level of power in the way of copper loss will be present only in the energised transformer. This will, in effect, give a differential temperature change, resulting in a resistance change between the two units, leading to unbalance errors similar to that in Figure 6.

Unless some form of thermal equalisation is introduced between the transformer pair a limit to size reduction could be set at some point prior to the normal balance limiting value. To a lesser degree this limitation will feature in the synchro isolation mode transformers.

It will be appreciated that the effect will be more pronounced with a synchro dwell at the extreme ends of the quadrant and have a diminishing effect towards the balanced mid quadrant positions.

\subsection{Winding and Core Characteristics}

Winding arrangements, to suit the various application requirements, will vary depending on the degree of resistance match required.

The winding technique must be such that, in effect, the cosine transformer presents half the winding resistance of the sine transformer with each side of the sine transformer equal about the centre tap. At the same time, the Scott Tee input turns relationship between both transformers must be accurately maintained. The need to match the winding resistance closely in the driver mode can be simplified by the use of bifilar or multifilar windings. Windings of this type may be highly capacitive which, though wholly acceptable in the low impedance state of the driver mode application, would be totally unacceptable in the monitor position.

As far as core materials are concerned, high nickel content iron, especially annealed nickel-iron, will surpass other material types in the way of linearity, with resultant low error factor insertion, and high effective shunt impedance. Limitations in available core flux swing values, however, leads to increased 
turns and increased winding resistance. This material is best suited to the requirements of the monitor application transformers.

Specially annealed 50-50 nickel-iron will give greater available core flux swing values, giving effectively reduced turns and winding resistance, lower values of shunt impedance and, to a lesser extent, increased angular error insertion. This material is more suited to the driver mode application, where low values of winding resistance are more important than high effective shunt impedance.

\subsection{Additional Design Characteristics}

Before leaving the subject of design in relation to transformed angular errors, it is of interest to note that with no size restrictions and access to special core materials, transformers can be designed with transformation ratio linearity, against variable excitation, to a few parts in a million, resulting in effective angular erros within a few seconds of arc.

As already emphasised, however, where weight and volume are the important criteria, a compromise must be struck on the question of transformer size related to the error factor, especially when the loaded device is considered. With this in mind, it might be advantageous to quantify the level of accuracy required in the transformer pair to meet an average mobile requirement.

This is demonstrated, in the following table, which relates the output voltage ratio requirements to a maximum allowable error factor of 0.6 minutes of arc at selected points over the first octant of synchro travel.

TABLE I

Output volts ratio requirements over first octant. 0.6 minutes of arc error.

Maximum angular error 0.6 minutes of arc, ratio $\frac{V_{o}(\sin )}{V_{o}(\cos )}$

\begin{tabular}{llll}
\hline $\begin{array}{l}\text { Synchro } \\
\text { angular } \\
\text { position } \\
\text { degrees }\end{array}$ & $\begin{array}{l}\text { True volts } \\
\text { ratio }\end{array}$ & $\begin{array}{l}\text { Allowable } \\
\text { variation each } \\
\text { side of true value }\end{array}$ & $\begin{array}{l}\text { \% variation of } \\
\text { true value }\end{array}$ \\
\hline 5 & 0.08749 & 0.00018 & 0.206 \\
15 & 0.26795 & 0.00019 & 0.071 \\
25 & 0.46631 & 0.00021 & 0.045 \\
35 & 0.70021 & 0.00026 & 0.037 \\
45 & 1.00000 & 0.00035 & 0.035 \\
\hline
\end{tabular}

It is of interest to note the apparent increase in allowable percentage variation about the true value towards the synchro zero position. This is characteristic across each quadrant, with minimum allowable variation at the mid quadrant and relaxed requirements towards the ends of the quadrant. This in effect counteracts the increase in errors due to variable transformation effects. If the $\mathbf{4 5}$ degree point is considered as the design centre in the particular example, the output voltage ratio will require to be between 1.00035 and 0.99965 .

Allowing an adequate design tolerance demands a turns ratio accuracy between the cosine and the sine input windings within the following limits of the nominal $3^{1 / 2} / 2$ value.

\begin{tabular}{lll}
\hline Nominal value & Upper limit & Lower limit \\
\hline 0.866025 & 0.866076 & 0.865974 \\
\hline
\end{tabular}

The values are, in the main, more stringent than most transformer application requirements. This, coupled with the need for consistent winding resistance balance, calls for an increased discipline in the way of core selection and winding control over the whole manufacturing cycle.

To ensure complete compatibility, transformers of this type are supplied in matched pairs and are designed to be used, with the relevant connections in either the sine or cosine mode presenting the required winding resistance balance, across the synchro lines. The use of a single design unit has distinct advantages. As well as the obvious interchangeability, a resolver mode application can be readily obtained, giving in effect a triple application capability.

The question of storage and procurement is also enhanced with the single transformer type.

Figure 7 shows, diagramatically, a Scott transformer pair arranged to facilitate both synchro application and resolver isolation mode of operation.

Transformers utilised in the synchro isolation mode are generally used in three main arrangements:-

(a) Three separate matched units.

(b) Two units in open delta connection.

(c) Two units in Tee-Tee connection.

Of the three methods the properly design Tee-Tee arrangement probably gives the best compromise between weight, cost and electrical performance, especially under loaded conditions. 


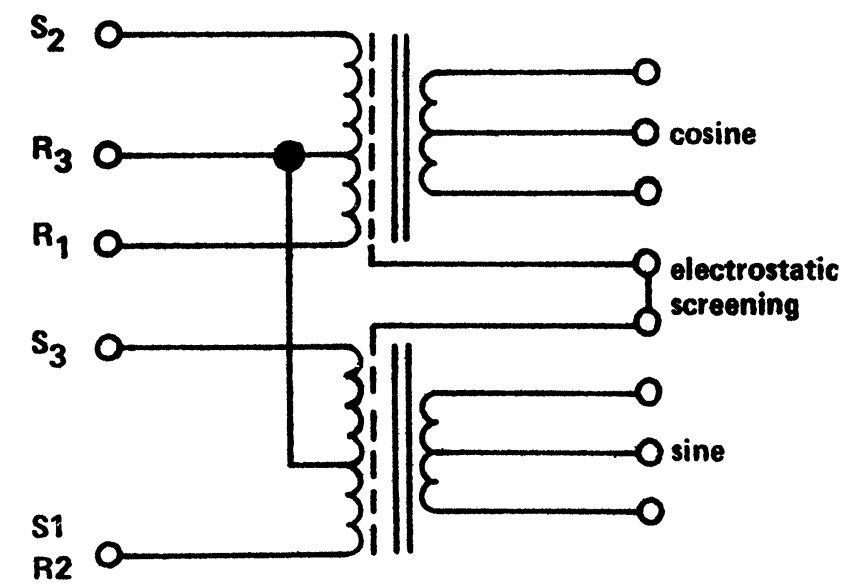

FIGURE 7 Transformers connected to give synchro or resolver isolation mode of operation.

\section{RELEVANT CALCULATIONS AND MEASUREMENTS}

The relationship between the winding resistance and the load resistance has been emphasised together with a method of angular error prediction at the mid quadrant points.

To illustrate the validity of the approach, two separate design types, representing in one case a synchro drive Scott pair and in the other case a monitor mode Scott pair, will be considered. The calculated mid quadrant error displacement will be compared with actual measured values of the driver mode arrangement.

If the sine transformer in each pair is taken as the reference, the voltage ratio through the transformer, synchro output to low impedance output, is 9:1 (90 volts line-to-line, 10 volts output) on both applications.

The driver pair is considered to look towards a line-to-line impedance of $4.86 \mathrm{~K}$ ohms. This, referred to the 10 volt winding, will be $60 \mathrm{ohms}$.

The monitor mode pair is loaded on both outputs with matched resistive loads of $10 \mathrm{~K}$ ohms.

The relevant resistance parameter of both pairs is as follows:-

\begin{tabular}{|c|c|c|c|c|c|}
\hline Pair & Application & $R_{L}$ & $R_{S}$ & $\begin{array}{l}R_{o s} \\
R_{o c}\end{array}$ & $\frac{R_{S}+R_{o s}^{\dagger}}{R_{L}}$ \\
\hline $\begin{array}{l}1 \\
2\end{array}$ & $\begin{array}{l}\text { Driver } \\
\text { Monitor }\end{array}$ & $\begin{array}{l}60 \\
10,000\end{array}$ & $\begin{array}{l}1.11 \\
20\end{array}$ & $\begin{array}{l}1.20 \\
25\end{array}$ & $\begin{array}{l}.042 \\
.0045\end{array}$ \\
\hline
\end{tabular}

†Winding to load resistance ratio.
The expression relating the winding resistance and effective load resistance to error factor $F$ was developed in 3.3 and for convenience is reproduced below.

$$
\frac{V_{o}(\sin )}{V_{o}(\cos )}=V_{o}(\tan )=\frac{1-\frac{R_{s}^{\prime}+R_{o s}}{R_{L}}}{1-\frac{4}{3} R_{L}\left(R_{2}^{\prime}+\frac{R_{s}^{\prime}}{4}\right)+\frac{R_{o c}}{R_{L}}}=F
$$

Inserting the values of transformer pair No. 1 and varying the degree of mismatch between the cosine and sine winding resistance values will give the following error displacement at the mid quadrant points.

TABLE II

Error factor, driver mode pair - varying $R_{2}^{\prime}$ with $R_{S}^{\prime}$ constant.

\begin{tabular}{clllll}
\hline \multicolumn{3}{c}{ Value increasing } & \multicolumn{3}{c}{ Value decreasing } \\
\hline \% variation & $F$ & $\begin{array}{l}\text { Error } \\
\text { minutes }\end{array}$ & $F$ & $\begin{array}{l}\text { Error } \\
\text { minutes }\end{array}$ \\
\hline 0.5 & 1.00006 & +.11 & .99994 & -.11 \\
1.0 & 1.00013 & +.22 & .99987 & -.22 \\
1.5 & 1.00019 & +.33 & .99981 & -.33 \\
2.0 & 1.00026 & +.44 & .99974 & -.44 \\
2.5 & 1.00032 & +.55 & .99968 & -.55 \\
3.0 & 1.00039 & +.66 & .99962 & -.66 \\
4.0 & 1.00051 & +.88 & .99949 & -.88 \\
5.0 & 1.00064 & +1.1 & .99936 & -1.1 \\
10.0 & 1.00128 & +2.2 & .99872 & -2.2 \\
20.0 & 1.00257 & +4.4 & .99743 & -4.4 \\
30.0 & 1.00386 & +6.6 & .99614 & -6.6 \\
\hline
\end{tabular}

As would be expected, the error values due to increasing the value of $R_{2}^{\prime}$ relative to $R_{s}^{\prime}$ has the same effect on the displacement as decreasing $R_{2}^{\prime}$, only the sign of the error is changed.

The positive error displacement against mismatch is plotted in Figure 8. Figure 9 shows the measured error form against various inserted values of increased $R_{2}^{\prime}$, over the first quadrant of synchro travel.

The increase and displacement of angular errors from the 45 degree null balanced resistance point, is clearly illustrated. Also to be noted is the apparent close agreement between the measured and calculated 45 degree points.

Similar calculations on the second transformer pair, will result in the following values (Only the positive values considered):

Compared with the drive mode transformer pair, the effect of the relatively lower value of winding to load resistance ratio, with the resultant relaxation of the winding resistance matching, is apparent. 


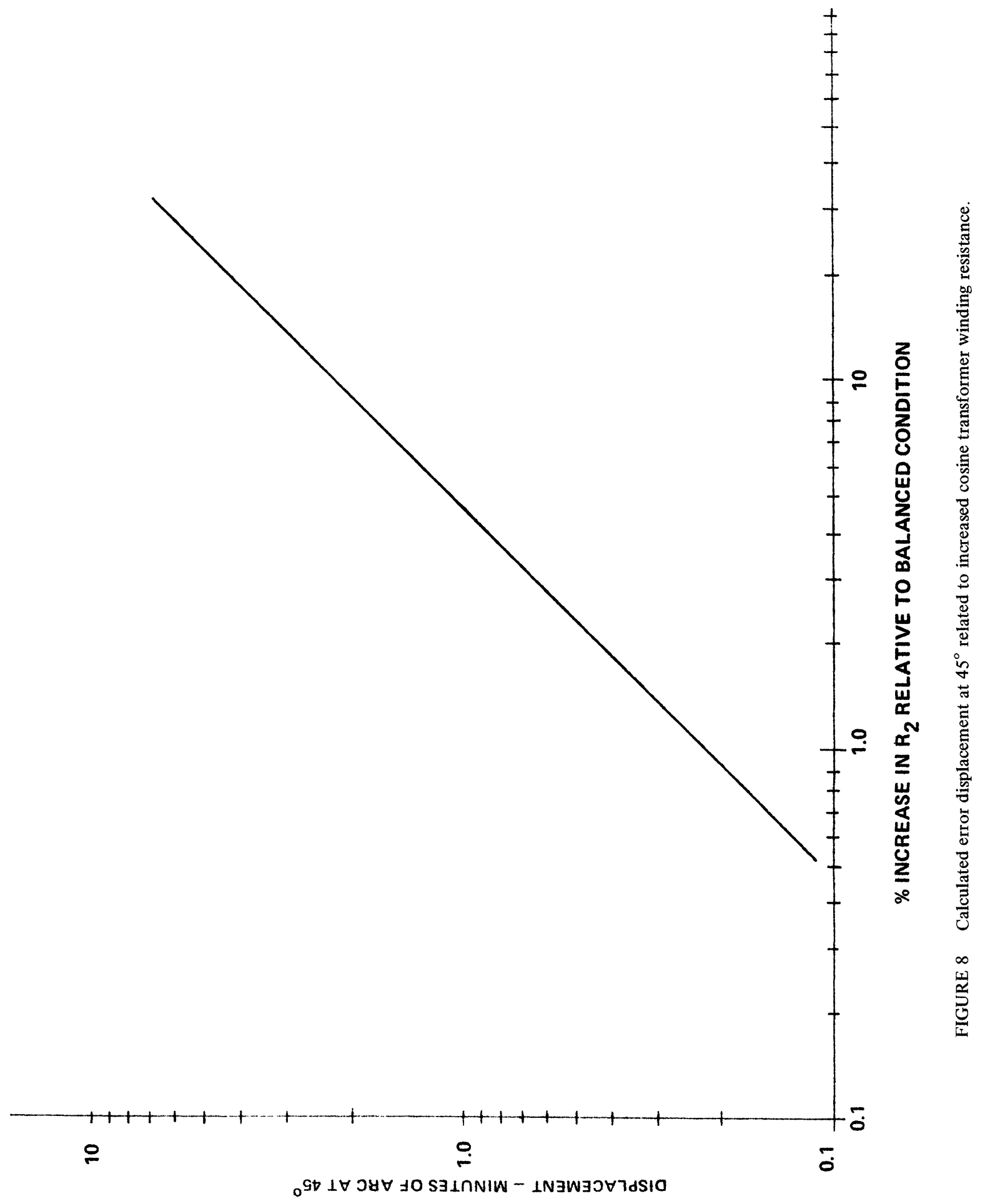




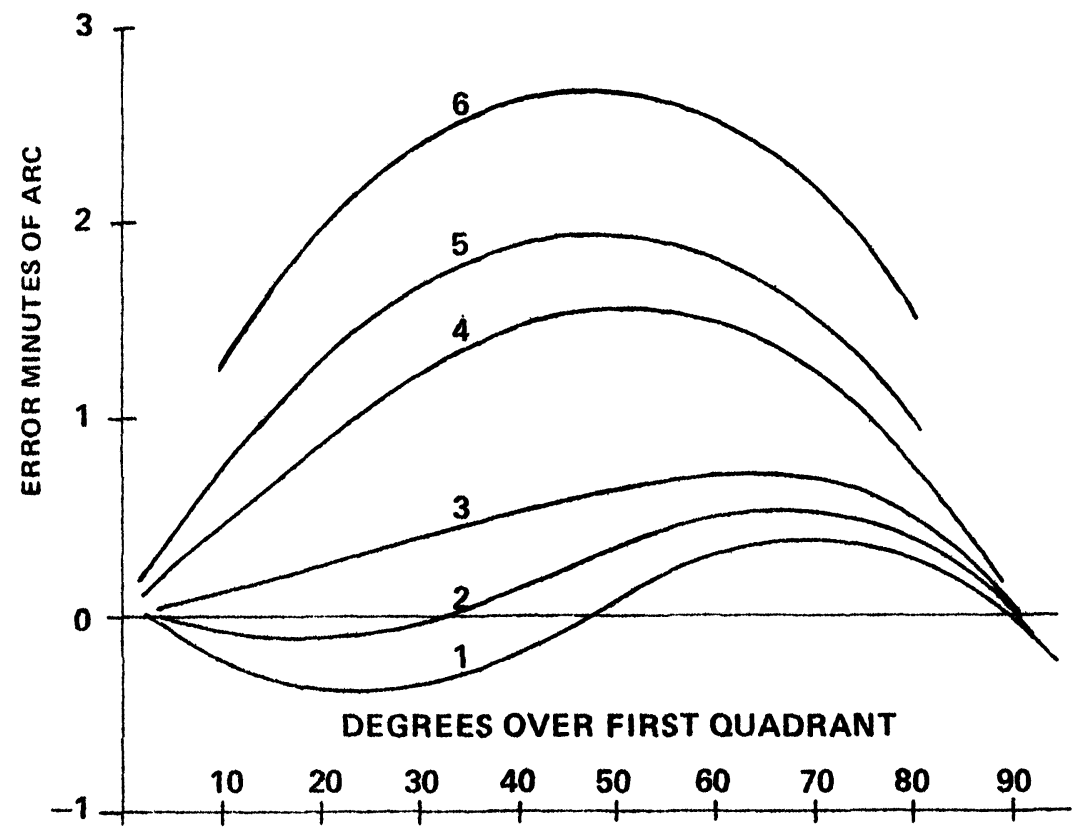

1 BALANCED RESISTANCE

$2 R_{2}$ INCREASED $1.2 \%$

$3 R_{2}$ INCREASED $2.6 \%$

$4 R_{2}$ INCREASED $7.7 \%$

$5 R_{2}$ INCREASED $9.5 \%$

$6 R_{2}$ INCREASED $13.0 \%$

FIGURE 9 Apparent transformation angular error form with various levels of mismatch between the sine and cosine input winding resistance - low impedance drive pair.

TABLE III

Error factor monitor mode pair - varying $R_{2}^{\prime}$ with $R_{S}^{\prime}$ constant

\begin{tabular}{cll}
\hline \% variation & $F$ & Error minutes \\
\hline 5 & 1.00006 & 0.11 \\
10 & 1.00013 & 0.23 \\
15 & 1.00020 & 0.34 \\
20 & 1.00027 & 0.46 \\
25 & 1.00033 & 0.57 \\
30 & 1.00040 & 0.69 \\
\hline
\end{tabular}

With transformer pairs working in this mode, only the transformation linearity and, to an extent, the input excitation currents, will be instrumental in the contribution of angular errors.

The measured transformed angular errors of a typical Scott transformer pair produced to the design considered is given in Figure 10.

\section{MECHANICAL AND ENVIRONMENTAL REQUIREMENTS}

In most mobile applications the ability to perform under conditions of malfunction and extreme environments will influence the translation of a given specification requirement, into a final hardware form.
This applies to all wound components, with an aggravation of the situation as the functional sophistication increases.

The requirements would include the ability to withstand at various severity levels:

(a) Climatic Exposure.

(b) Vibration.

(c) Mechnical Shock.

(d) High and Low Temperatures.

(e) Thermal Shock.

Additional functional requirements, such as the ability to perform after severe continuous overloading, may be required.

The ability to meet the climatic conditions will be influenced by the severity demanded., this in turn influences the type of protection in the way of utilisation of process methods.

Protective impregnating, and encapsulating materials, could vary from solventless varnishes to carefully blended epoxy resins, with special filling agent. Impregnating material must be carefully chosen in the presence of high nickel content core materials. Incompatibility can cause degredation of the magnetic characteristics, giving instability, especially with temperature variation. 


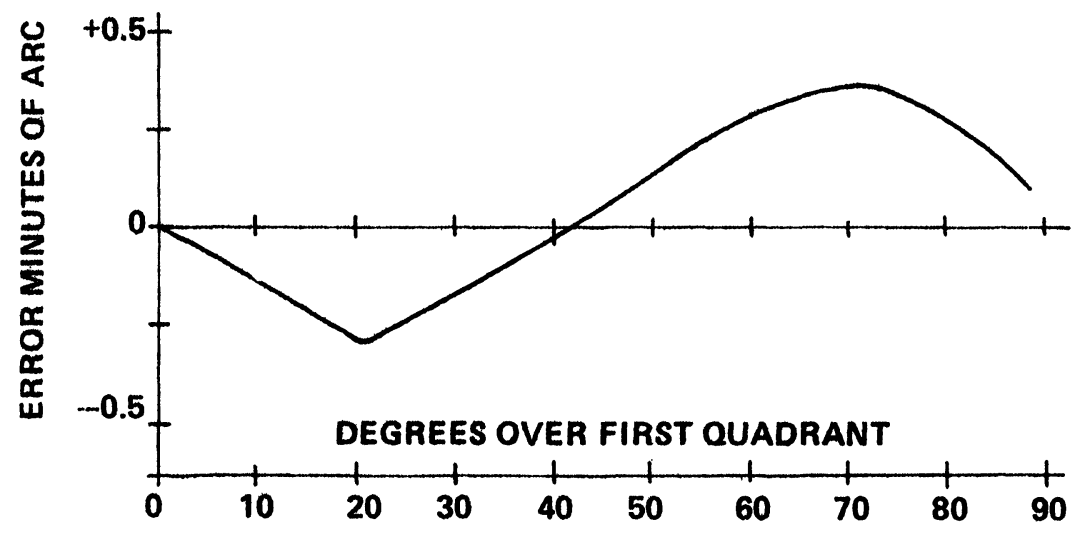

FIGURE 10 Typical angular error form due to transformation - high impedance monitor mode Scott pair

The mechanical aspects would, related to the severity levels demanded, take into consideration such things as the method of fixing and terminations. The effect of shock on core locking materials and the ability of encapsulants, such as epoxies, to withstand rapid changs of temperatures, would also merit scrutiny.

Malfunction in the way of severe overloading cannot be ruled out. Synchro lines, especially in shipborne systems, are vulnerable to shorting out. Under conditions such as this, transformers driving synchro elements, may require to work into sustained overloads several times the working values with the ability to function normally after a recovery period.

\section{CONCLUSIONS}

A design approach based on equalisation of series winding resistance, selection of core material and a level of control at the manufacturing stages, will lead to a reduction of size and error insertion in the transformer application considered.

Balanced winding resistance has the added advantage of allowing a reduction of the tolerance requirements of the load resistors in the monitor role application.

The relationship between, resistance balance and acceptable hardware volume and weight, is more apparent in mobile systems, especially when the transformers look towards low impedance synchro loads.

The limitation of size reduction due to the effective resistance, unit-to-unit divergence would be minimised, with a high degree of thermal equalisation between both units in the Scott pair. It is possible this could be best achieved with toroidally wound transformers assembled in a single package, having good thermal contact between both units in the package, and the utilisation of an encapsulating material with a high thermal conductivity.

\section{ACKNOWLEDGEMENT}

The author would like to acknowledge the overall assistance given by the Ferranti Digital Systems Department, Bracknell, particularly Mr. A. R. Wooldridge for his advice on the concept of the winding resistance balance theory and his subsequent technical considerations.

\section{REFERENCES}

1. M. Pratt, Contributed paper at the Institution of Electronic and Radio Engineers Colloquium on "Small Power Transformers and Chokes" held at the I.E.R.E. Headquarters on 10th April, 1974.

2. M. Pratt, "The Use of Matched Transformers in synchrodigital converters" Electronics and Power, 20, 7, 284-288, 1974. 

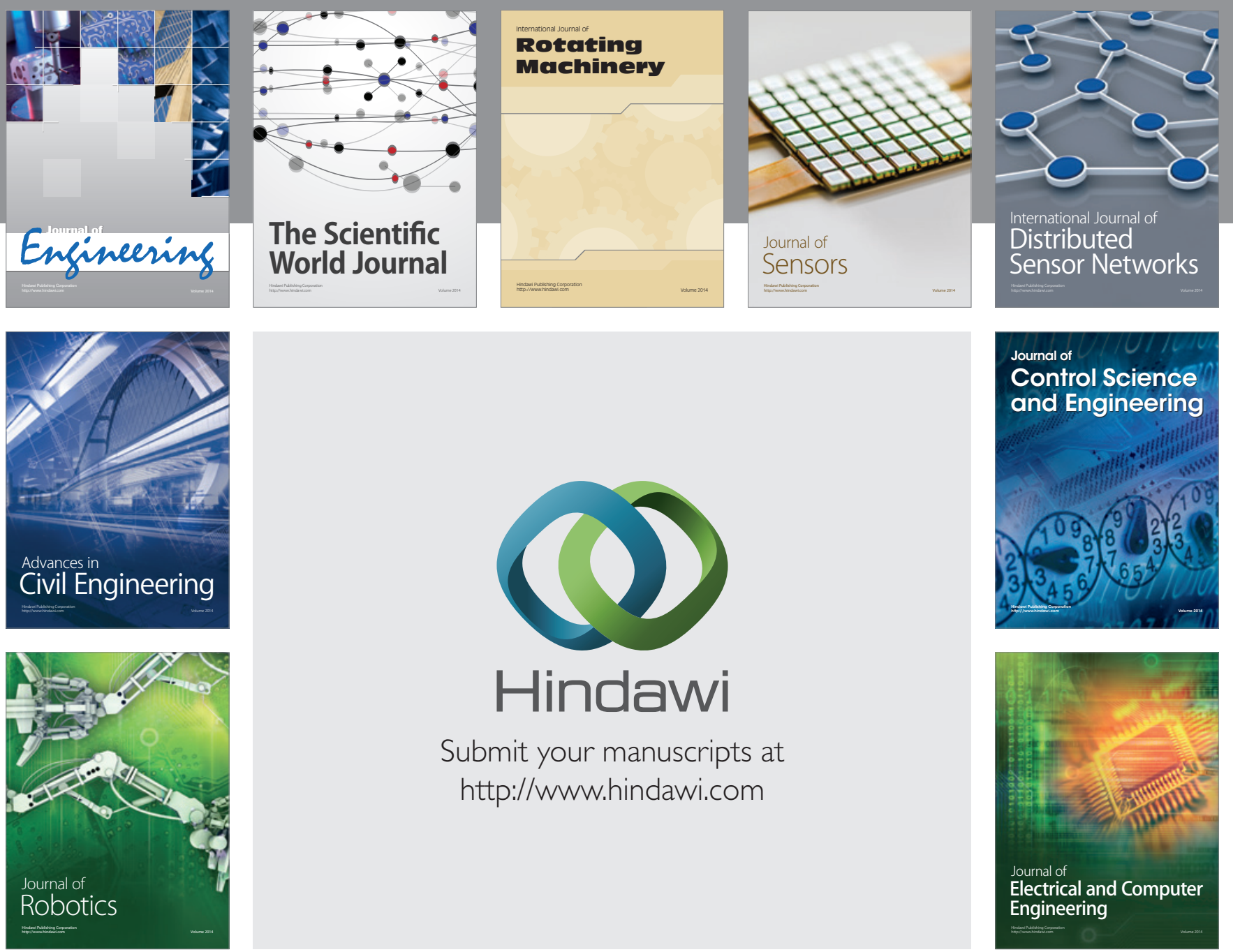

Submit your manuscripts at

http://www.hindawi.com
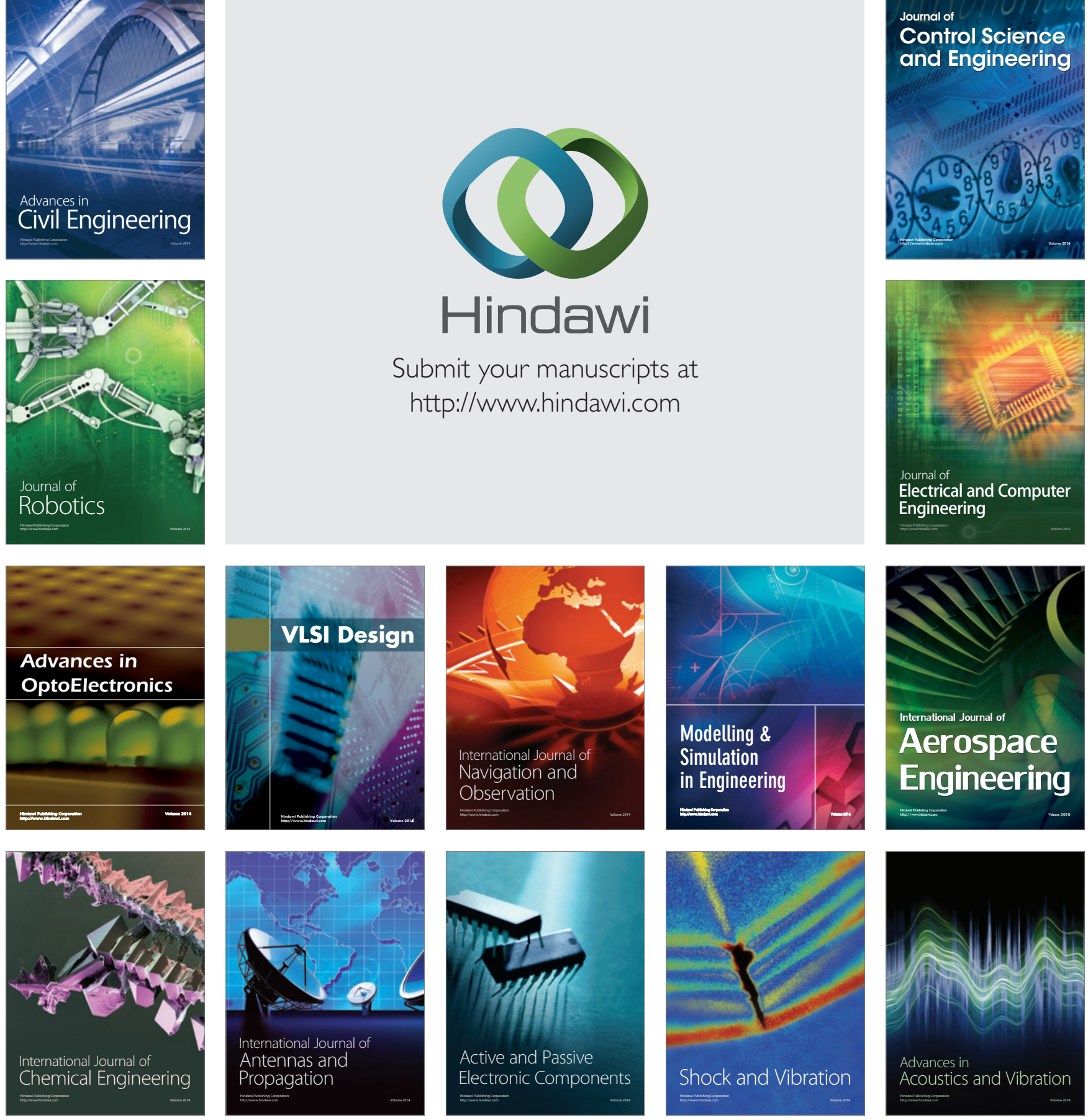